PII S1359-6462(97)00124-3 1359-6462/97\$17.00+.00

\title{
THE CHEMICAL DRIVING FORCE FOR RAFTING IN SUPERALLOYS
}

\author{
F.R.N. Nabarro \\ Division of Materials Science and Technology, \\ C.S.I.R., P.O.Box 395, Pretoria 0001 \\ Condensed Matter Physics Research Unit, \\ University of the Witwatersrand, Private Bag 3, WITS 2050, \\ Johannesburg, South Africa
}

(Received February 13, 1997)

(Accepted February 25, 1997)

Until recently, all theories of the driving force for rafting have considered the compositions of the two phases to be fixed, although accepting that the rate of rafting might be controlled by diffusion.

The analysis in the elastic regime (e.g. 1,2) rests on Eshelby's recognition that the thermodynamic pressure on an interface has two components: (i) the difference in elastic energy densities across the interface, and (ii) the work done by the normal traction across the interface when the interface moves.

Suppose a tensile stress $\sigma$ is applied along a cube axis to a superalloy with a positive misfit, i.e the lattice parameter of the $\gamma^{\prime}$ particles exceeds that of the $\gamma$ matrix. There is a large increase in the energy density in the $\gamma$ channels parallel to the applied tensile stress, because the material already has an internal stress in the same direction. There is also a first order increase in the work done by the traction across the transverse interfaces. These effects both cause a thermodynamic pressure, tending to displace the corresponding interface from $\gamma^{\prime}$ towards $\gamma$. If the elastic constants of $\gamma$ and $\gamma^{\prime}$ are equal, the two pressures are equal, and there is no tendency to rafting. If $\gamma^{\prime}$ has a higher elastic modulus, the elongation of the $\gamma$ sheets parallel to the tensile axis is constrained, the additional thermodynamic pressure on the lateral interfaces is reduced, and rafting occurs by outward displacement of the transverse interfaces.

When plastic flow occurs, the difference in elastic constants becomes negligible. A high energy density builds up in the transverse $\gamma$ sheets, and rafting occurs by outward motion of the transverse interfaces, reducing the volume which has a high energy density.

Recently, Saito et al. (3) outlined a totally different model of the driving force for rafting. When an external stress is applied to a superalloy, the changes in elastic energy density in the $\gamma$ particles, in the transverse $\gamma$ sheets and in the lateral $\gamma$ sheets are all different: (Saito et al. assume "that elastic strain energy increases and decreases in the $\gamma$ matrix channels parallel and perpendicular to the tensile stress, respectively, and that elastic strain energy has practically no change in the $\gamma^{\prime}$ precipitate phase". If this refers to the elastic regime, it would imply a positive misfit, which seems unlikely, especially since the alloy shows normal rafting.) In the plastic regime, the large stresses build up in the transverse $\gamma$ sheets. This change in elastic energy density produces a relative vertical shift of the free-energy versus com- 


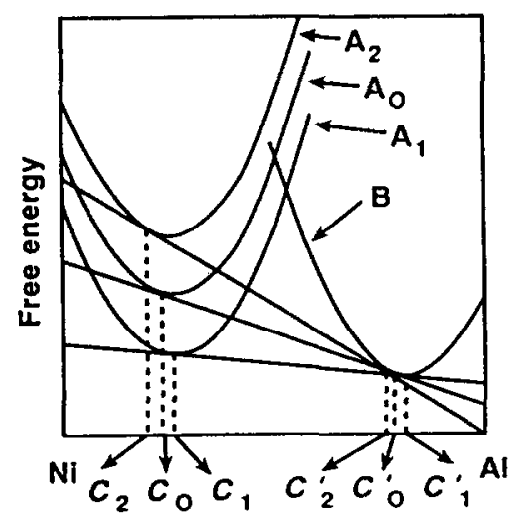

Figure 1. Schematic free energy diagram of $\gamma(A)$ and $\gamma(B)$ phases. $A_{0}$ is before loading, and $A_{1}$ and $A_{2}$ are perpendicular to the stress and parallel to the stress, respectively. Al equilibium concentrations are changed from $C_{0}$ to $C_{2}$ in $\gamma$ and $C_{0}^{\prime}$ to $C_{2}^{\prime}$ in $\gamma^{\prime}$ near an interface parallel to the stress, from $C_{0}$ to $C_{1}$ in $\gamma$ and $C_{0}^{\prime}$ to $C^{\prime}$, near an interface perpendicular to the stress, respectively. (Reproduced from ref. (3)).

position curves of the two phases, with a consequent shift of the compositions and proportions of the two phases which are in equilibrium. They illustrate this with the diagram of Fig. 1.

We may develop this idea in more detail by making some simplifying assumptions.

Our analysis considers only the change in enthalpy between two states, one in which the two phases have the compositions which are in equilibrium in the absence of external stress, the extemal stress has been applied, but no diffusion has occurred, and one in which the two phases have the homogeneous compositions which are in equilibrium under the applied stress. We do not attempt to treat the intermediate configuration in which some diffusion has occurred, but the compositions of the phases are inhomogeneous.

Suppose that one of the phases has a very sharp free-energy/composition curve, so that it is virtually a line compound of composition $c^{\prime}$ and free energy per unit volume $U_{0}$ We shall take this to represent $\gamma$, and, following Saito et al., neglect the dependence on applied stress of the free energy of

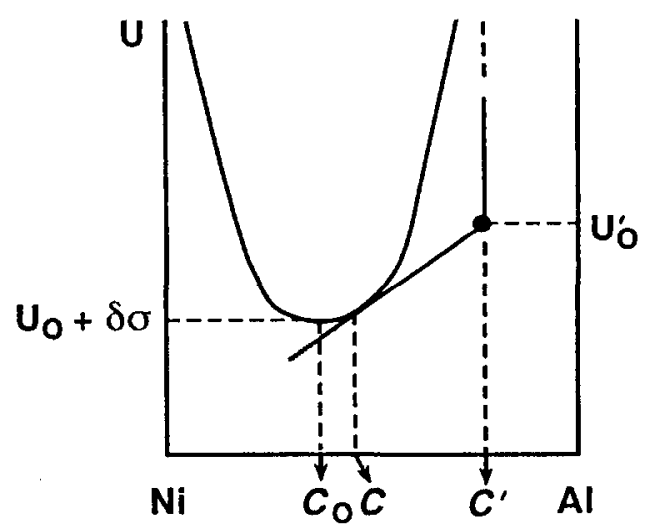

Figure 2. Enthalpy as a function of composition. For the $\gamma$ phase the enthalpy is parabolic, with a minimum at $c_{0}$ and a vertical displacement proportional to the applied stress $\sigma$. The $\gamma$ phase has a fixed composition $c^{\prime}$ with fixed enthalpy $\dot{U}_{0}$. 


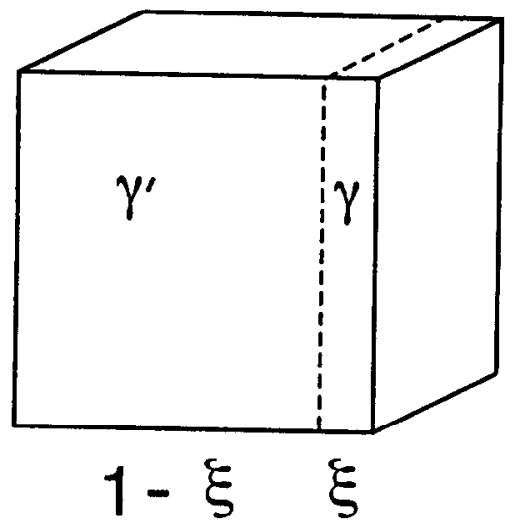

Figure 3. The unit cube divided by a single interface into volumes $\xi$ of $\gamma$ phase and $1-\xi$ of $\gamma^{\prime}$ phase.

this phase. The other phase, which we shall take to represent $\gamma$, has, under tensile stress $\sigma$, composition $c$ and free energy per unit volume

$$
U_{\gamma}=U_{o}+K\left(c-c_{o}\right)^{2}+\delta \sigma
$$

as is represented in Fig. 2.

We shall consider only terms linear in $\sigma$. We take the simplified model of a unit cube divided by a boundary parallel to the tensile axis into a volume $\xi$ of the $\gamma$ phase and $1-\xi$ of the $\gamma^{\prime}$ phase (Fig. 3 ).

The enthalpy of the unit cube is

$$
\begin{aligned}
& H=\xi U \gamma+(1-\xi) U_{\gamma^{\prime}}-(1-\xi) \delta \sigma \\
& =\xi\left[U_{o}+K\left(c-c_{o}\right)^{2}+\delta \sigma\right]+(1-\xi) U_{o}^{\prime}-(1-\xi) \delta \sigma
\end{aligned}
$$

Let the overall composition of the alloy be $c_{\mathrm{n}}$. Then

$$
\xi c+(1-\xi) c^{\prime}=c_{a}
$$

or

$$
c=c^{\prime}-\frac{c^{\prime}-c_{a}}{\xi}
$$

and

$$
H=\xi\left[U_{o}-U_{o}^{\prime}+2 \delta \sigma+K\left(c^{\prime}-c_{o}-\frac{c^{\prime}-c_{a}}{\xi}\right)^{2}\right]+U_{o}^{\prime}-\delta \sigma
$$


We have equilibrium when

$$
\partial H / \partial \xi=0
$$

or

$$
\begin{gathered}
\xi=\left[\frac{K^{2}\left(c^{\prime}-c_{a}\right)^{2}}{S^{2}+2 K \delta \sigma}\right]^{1 / 2} \\
\approx \frac{K\left(c^{\prime}-c_{a}\right)}{S}\left(1-\frac{K \delta \sigma}{S^{2}}\right),
\end{gathered}
$$

where

$$
S=\left[K\left(U_{o}-U_{o}^{\prime}\right)+K^{2}\left(c^{\prime}-c_{o}\right)^{2}\right]^{1 / 2}
$$

The significance of $S$ is that $S^{2} / K$ is the excess of the free energy of $\gamma$ over that of $\gamma$ when $\gamma$ has the composition of $\gamma^{\prime}$.

When stress is applied but no adjustment of composition has occurred,

$$
\xi=\frac{K\left(c^{\prime}-c_{a}\right)}{S}
$$

Since, in the final state, $\partial H / \partial \xi=0$, the change of $\xi$ from the value (7) to the value (5) increases $H$ by

$$
\begin{aligned}
\Delta H & =\frac{1}{2}\left[\frac{K^{2}\left(c^{\prime}-c_{a}\right) \delta \sigma}{S^{2}}\right]^{2} \frac{\partial^{2} H}{\partial \xi^{2}} \\
& =\frac{K^{2}\left(c^{\prime}-c_{a}\right) \delta^{2} \sigma^{2}}{S^{3}}
\end{aligned}
$$

The value of $\partial^{2} H / \partial \xi^{2}$ should be evaluated in the final state, but, since we shall show that the changes in composition are small, we may replace this value by its value in the initial state.

This change in enthalpy is achieved during a displacement

$$
\Delta \xi=-\frac{K^{2}\left(c^{\prime}-c_{a}\right) \delta \sigma}{s^{3}},
$$

and so the initial chemical thermodynamic pressure on the interface is

$$
P_{\mathrm{c}}=2 \delta \sigma
$$


This may be compared with the thermodynamic pressure arising from purely elastic effects, which is of order

$$
P_{\mathrm{e}}=m \delta \sigma,
$$

where $m$ is the fractional difference of the elastic constants of $\gamma$ and $\gamma^{\prime}$. Since $m$ is usually of order 0.1 , $P_{\mathrm{c}}$ is substantially greater than $P_{\mathrm{e}}$. On the other hand, $P_{\mathrm{c}}$ remains roughly constant as the interface migrates, while $P_{c}$ decreases to zero at the value of $\Delta \xi$ given by (9). Since $K$ and $S$ are energies related to the phase diagram, we expect them both to be of order $R T$ per mole or $1.6 \times 10^{5} R T \mathrm{~m}^{-3}=1.3 \times 10^{9}$ $\mathrm{Jm}^{-3}$, while $c^{\prime}-\mathrm{c}_{\mathrm{a}} \approx 0.1, \delta \approx 3 \times 10^{-3}$, and $\sigma \approx 500 \mathrm{Mpa}=5 \times 10^{8} \mathrm{JM}^{-3}$. Then $|\Delta \xi|=10^{-4}$, and the chemical driving force is exhausted before any measurable change in the shape of the particles has occurred.

\section{References}

1. F.R.N. Nabarro, Met. Trans. A 27A 513 (1996).

2. F.R.N. Nabarrio, C.M. Cress and P. Kotschy, Acta Mater. 44, 3189 (1996).

3. M. Saito, T. Aoyama, K. Hidaka, H. Tamaki, T. Ohashi, S. Nakamuro and T. Suzuki, Scripta Mater. 34,1189 (1996). 